\title{
An infant case of renovascular hypertension in moyamoya disease treated by angioplasty
}

\author{
Jianming Guo ${ }^{1}$, Lianrui Guo ${ }^{1}$, Gao Zeng ${ }^{2}$, Zhu Tong ${ }^{1}$, Xixiang Gao ${ }^{1}$, Yongquan $\mathrm{Gu}^{1}$ \\ Departments of ${ }^{1}$ Vascular Surgery and ${ }^{2}$ Neurosurgery, Xuanwu Hospital, Capital Medical University; Institute of Vascular \\ Surgery, Capital Medical University, Beijing, China. \\ E-mail: gu15901598209@gmail.com \\ Received: 9th May 2017, Revised: 1st July 2017, Accepted: 10th July 2017
}

\begin{abstract}
SUMMARY: Guo J, Guo L, Zeng G, Tong Z, Gao X, Gu Y. An infant case of renovascular hypertension in moyamoya disease treated by angioplasty. Turk J Pediatr 2018; 60: 331-334.

Here we report the treatment experience of an 18-month-old female infant case of renovascular hypertension due to moyamoya disease by angioplasty. Although renal blood flow was recovered immediately after operation, 12 months follow-up found severe restenosis which required us to perform a right renal autotransplantation.
\end{abstract}

Key words: renovascular hypertension, moyamoya disease, angioplasty.

Moyamoya disease (MMD) is a chronic cerebrovascular disorder of unknown pathogenesis in which the supraclinoid internal carotid arteries exhibit progressive occlusion and tortuous arterial collateral vessels form at the base of the brain to serve as the distal branches of the cerebral circulation. ${ }^{1}$ Although very rare, renal artery lesions in MMD have been described in several recent case reports. ${ }^{2-6}$ Three cases of renovascular hypertension (RVHT) in MMD treated by angioplasty were published in 1988 and 2000.3,7 Notably, the patients in these cases were adults, and in this case report, we present the first report of an infant with MMD and RVHT successfully treated by angioplasty based on 12 months of follow-up observation.

\section{Case Report}

An 18-month-old female infant presented in February 2014 with a 1-month history of dysphoria. The patient had no history of trauma, and her personal and family histories were unremarkable. On physical examination, the infant's blood pressure was 240/170 $\mathrm{mmHg}$, and the following antihypertensive agents were administered: metoprolol tartrate (Vetter Pharma-Fertigung GmbH \& Co. KG. Ravensburg, Germany) 25 mg b.i.d. No bleeding or infarction was identified by cranial computed tomography (CT). Internal carotid arteriography revealed narrowing of the internal carotid artery and complete occlusion of the anterior and middle cerebral arteries. Profuse tortuous basal collateral vessels, so-called moyamoya vessels, also were observed. Peripheral renin activity was found to be $12.2 \mathrm{ng} / \mathrm{ml} / \mathrm{hr}$ (reference ranges: $0.29-3.7 \mathrm{ng} / \mathrm{ml} / \mathrm{hr}$ ), and renal vascular ultrasound showed that the right kidney was small in size, the renal artery velocity was $248 \mathrm{~cm} / \mathrm{s}$, and the resistive index (RI) was 0.54. After hospital admission, the infant's blood pressure was measured at least four times per day.

Angioplasty was performed while the patient was under general anesthesia. The procedure was performed through a right femoral artery approach and a 5F sheath was inserted. Aortic angiography showed occlusion of the left renal lower pole artery, normal flow in the left renal upper pole artery, atrophy in the left renal region, and severe stenosis in the main trunk of the right renal artery (Fig. 1). A Cobra 2 (Cordis, Johnson \& Johnson, Chihuahua, CP, Mexico) catheter was used to access the right renal artery, and the guidewire was changed to an 0.018-inch, 200-cm guidewire used for 2-15 $\mathrm{mm}$ and 3.5-15 mm balloon (Ryujin Plus, Terumo Co, Fujinomiya, Japan) inflation. On follow-up angiography, the treated artery appeared to have improved considerably (Fig. 2 ). No operative complications occurred, and 
subsequent renal ultrasound showed excellent blood flow in the kidney. On postoperative day 5 , the patient was discharged home with a blood pressure of 130/80 $\mathrm{mmHg}$ controlled by metoprolol tartrate (Vetter Pharma-Fertigung GmbH \& Co. KG. Ravensburg, Germany) 12.5 mg b.i.d. At the 6-month follow-up, ultrasound revealed moderate restenosis, and at 12 months, the infant was asymptomatic with a blood pressure of $200 / 140 \mathrm{mmHg}$ despite long-

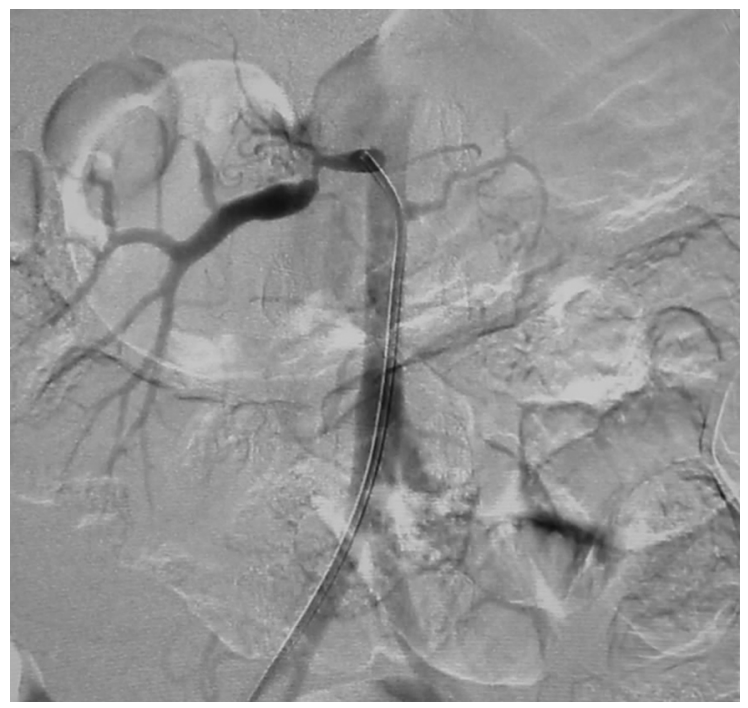

Fig. 1. Aortagram showing stenosis in the main trunk of the right renal artery.

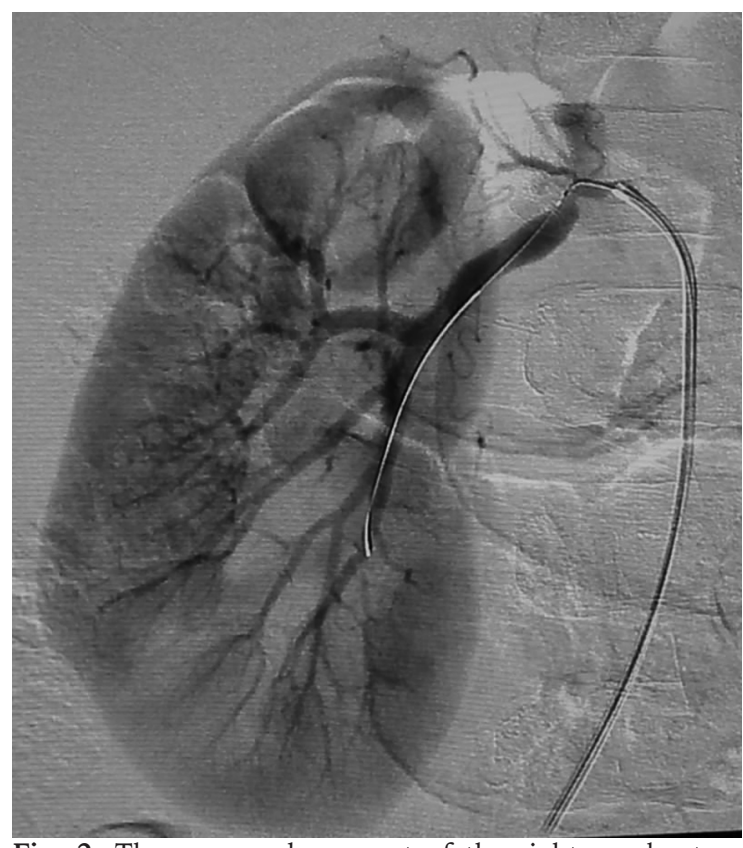

Fig. 2. The narrowed segment of the right renal artery was dilated after balloon angioplasty, with no evidence of residual stenosis. term maintenance therapy with metoprolol tartrate. CT showed obvious restenosis in the right renal artery (Fig. 3), and the patient underwent right renal autotransplantation. In left fossa iliaca, renal and external iliac artery, and renal and external iliac vein, anastomoses were performed using end-to-side technique separately. The diameter of venous anastomosis was $1.0 \mathrm{~cm}$ and arterial anastomosis was 0.6 $\mathrm{cm}$. No operative complications occurred, and the recovery was uneventful with improvement of the RVHT observed. Before writing this article, an informed consent was received from the family.

\section{Discussion}

The first case of moyamoya arteriopathy was described in Japan by Takeuchi and Shimizu ${ }^{8}$ in 1957, and since then only a few sporadic cases have been reported worldwide, mainly in case reports or as retrospective series. Thus, clinical data for this condition remain inadequate, with long-term follow-up results remaining unavailable. The typical appearance of small, fragile, basal, collateral vessels on angiography prompted Suzuki and Takaku ${ }^{9}$ to describe the vasculopathy using the Japanese word "moyamoya," which means "hazy, cloudy, or

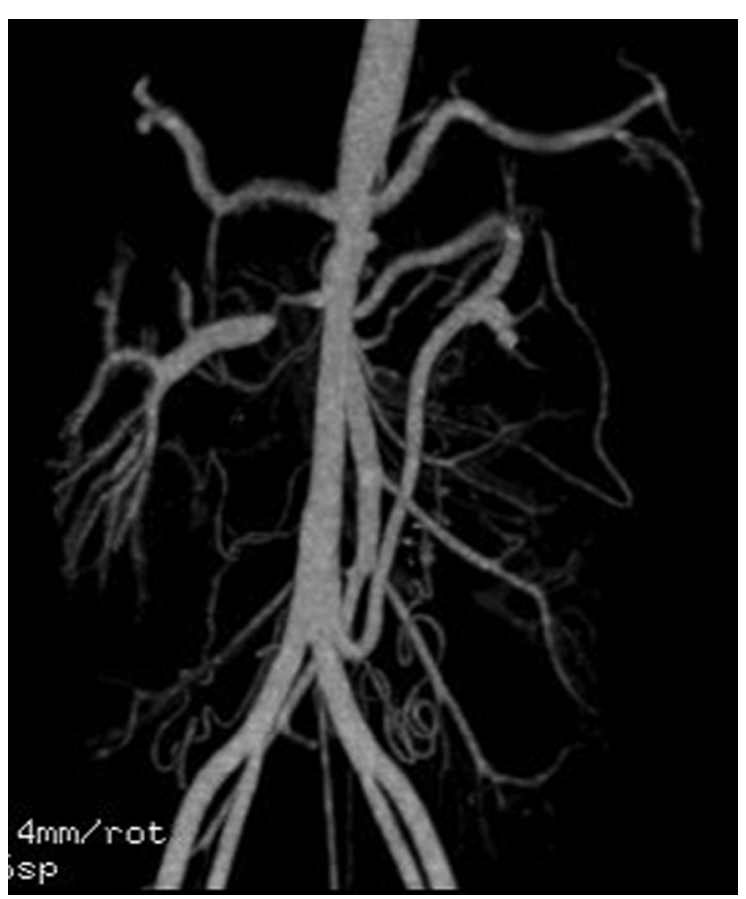

Fig. 3. Angiograph taken at the 12-month follow-up showing obvious restenosis in the right renal artery. 
puff of smoke." Pathologic studies have shown that obstructive lesions in the cerebral vessels are caused primarily by fibrous thickening of the intima and a small amount of lipid deposition. The internal elastic lamina is well preserved, and no significant inflammatory cell infiltration has been noted in the vascular walls. ${ }^{10}$ Ikeda $^{11}$ reported that the histopathologic and morphometric characteristics of extracranial vessels are similar to those of the intracranial vessels.

The etiology of the disorder is unclear, and most reported cases occurred in Asia predominantly in female children. Co-existence of cerebral and renal lesions has been rarely reported. The prevalence of renal artery stenosis in patients with MMD is $5 \%-7 \%$, Togao et al. ${ }^{2}$ and Yamada et $\mathrm{al}^{3}$. reported an incidence of RVHT in MMD of $3 \%-7 \%$. These studies also revealed that renal artery stenosis tended to be located in the proximal region of the main branch, an observation consistent with findings in the present case. In the absence of brain ischemia manifestation, differentiating atherosclerosis from Takayasu's arteritis by angiographic appearance alone is difficult.

As endovascular therapy for RVHT in children due to fibromuscular dysplasia (FMD) and Takayasu arteritis, Colyer et al. ${ }^{12}$ and Radanović et al. ${ }^{13}$ revealed that angioplasty is technically and clinically feasible and safe. Novick et al. ${ }^{14}$ found that autotransplantation and repair with a saphenous vein patch constitute an effective approach to renal artery repair when branch arterial reconstruction is required. Due to the rarity of this disease, the effectiveness of any medication or procedure for RVHT in MMD patients remains controversial, and close followup is required.

In 1997, Choi et al. ${ }^{6}$ reported a case of renal artery stenosis in a 16-month-old female MMD patient. The infant's blood pressure and kidney size were both normal, and the patient was treated by right renal angioplasty. Although the case did not include results of regular follow-up, hypertension was detected in the patient at the age of 8 years 5 months, when the patient was readmitted for encephaloduroarteriosynangiosis. Considering that stenotic lesions in MMD are usually progressive, for patients with MMD combined with hypertension, early detection of renal artery lesions and close follow-up are critical. Because hypertension was not originally detected in the patient reported by Choi et al. ${ }^{6}$, their report cannot provide information regarding the therapeutic effect of angioplasty for RVHT. Among the three adult cases of RVHT in MMD treated by angioplasty described in the literature ${ }^{3,7}$, all showed immediate improvement in blood pressure post-procedure, but no follow-up data were provided. Thus, based on searches of the PubMed and Ovid MEDLINE databases, the present report seems to be the first of an infant with MMD and RVHT and the first to describe outcomes of 12 months of follow-up. With the recurrence of hypertension and restenosis of the renal artery after 12 months in the present case, more cases need to be studied in order to determine the effectiveness of the treatment approach applied.

In conclusion, the present case suggests that angioplasty is likely a safe and reliable treatment approach for RVHT in patients with MMD, but it may not bring long-term blood pressure improvement. More clinical cases of this rare disease need to be followed.

\section{Acknowledgement}

This work is supported by Grant No. XMLX201610 from the Beijing municipal administration of hospitals clinical technology innovation program, Grant No. DFL20150801 from the Beijing municipal administration of hospitals climbing talent training program and Grant No.2016000020124G108 from the Beijing outstanding talents project.

\section{REFERENCES}

1. Jea A, Smith ER, Robertson R, Scott RM. Moyamoya syndrome associated with Down syndrome: outcome after surgical revascularization. Pediatrics 2005; 116: e694-e701.

2. Togao O, Mihara F, Yoshiura T, et al. Prevalence of stenoocclusive lesions in the renal and abdominal arteries in moyamoya disease. AJR Am J Roentgenol 2004; 183: 119-122.

3. Yamada I, Himeno Y, Matsushima Y. Renal artery lesions in patients with moyamoya disease: angiographic findings. Stroke 2000; 31: 733-737.

4. Ahmed J, Ali US. Moyamoya disease with renal artery and external iliac artery stenosis. Indian J Pediatr 2011; 78: 99-102.

5. Shoskes DA, Novick AC. Surgical treatment of renovascular hypertension in moyamoya disease: case report and review of the literature. J Urol 1995; 153: 450-452. 
6. Choi Y, Kang BC, Kim KJ, et al. Renovascular hypertension in children with moyamoya disease. $\mathrm{J}$ Pediatr 1997; 131: 258-263.

7. Halley SE, White WB, Ramsby GR, Voytovich AE. Renovascular hypertension in moyamoya syndrome. Therapeutic response to percutaneous transluminal angioplasty. Am J Hypertens 1988; 1: 348-352.

8. Takeuchi K, Shimizu K. Hypoplasia of the bilateral internal carotid arteries. Brain Nerve (Tokyo) 1957; 9: $37-43$.

9. Suzuki J, Takaku A. Cerebrovascular "moyamoya" disease: a disease showing abnormal net-like vessels in base of brain. Arch Neurol 1969; 20: 288-299.

10. Nishimoto A, Takeuchi S. Abnormal cerebrovascular network related to the internal carotid arteries. J Neurosurg 1968; 29: 255-260.
11. Ikeda E. Systemic vascular changes in spontaneous occlusion of the circle of Willis. Stroke 1991; 22: 1358-1362.

12. Colyer JH, Ratnayaka K, Slack MC, Kanter JP. Renal artery stenosis in children: therapeutic percutaneous balloon and stent angioplasty. Pediatr Nephrol 2014; 29: $1067-1074$.

13. Radanović B, Cacić Z, Perkov D, Smiljanić R, Corić SR, Ilakovac K. Endovascular therapy of renovascular hypertension in children: single center analysis. Eur J Pediatr Surg 2009; 19: 135-140.

14. Novick AC, Jackson CL, Straffon RA. The role of renal autotransplantation in complex urological reconstruction. J Urol 1990; 143: 452-457. 